\title{
Successful Cessation Programs that Reduce Comorbidity may Explain Surprisingly Low Smoking Rates among Hospitalized COVID-19 Patients
}

\author{
Bruce Cohen ${ }^{1}$, Aaron Nichols ${ }^{1}$, Stephen Grant ${ }^{1}$, Zack Blumenfeld ${ }^{1}$, Dennis Dougherty ${ }^{1}$, R. Michael Alvarez ${ }^{1}$, Beate Ritz ${ }^{2}$, \\ Henry A. Lester ${ }^{1}$
}

1 California Institute of Technology

2 University of California, Los Angeles

Funding: The author(s) received no specific funding for this work.

Potential competing interests: The author(s) declared that no potential competing interests exist.

\section{Abstract}

Recent, non-peer-reviewed reports and meta-analyses suggest that smoking may reduce the risk of hospitalization with COVID-19, because the prevalence of smoking among hospitalized COVID-19 is less than that of the general population. However, there are two alternative classes of explanations for this phenomenon. Class (1) is the failure to report, or to accurately record, smoking history during emergency hospital admissions and other interviews. Face-toface interviews can introduce bias into the responses to attitudinal and behavioral questions not present in the selfcompletion interviews typically used to measure smoking prevalence in the general population. Subjects in face-to-face interviews may be unwilling to admit socially undesirable behavior and attitudes under direct questioning. For example, urine testing of hospitalized patients for cotinine showed that smokers were under-counted by $37 \%$ because incoming patients failed to inform staff about their smoking behavior. Class (2), various types of "reverse" causation, including a pre-disposition to avoid smoking among COVID-19 patients with tobacco-related comorbidities, may also contribute to the difference between smoking prevalence in the COVID-19 and general population. Patient cohorts hospitalized with COVID-19 may be less prone to use tobacco than the general population. A potentially robust "reverse causation" hypothesis for reduced prevalence of smokers in the COVID-19 population is enrichment of patients in the population with serious comorbidities that have previously motivated them to quit smoking. We ask whether this "smoking cessation" mechanism accounts for a detectable fraction of the reduced prevalence of smokers in the COVID-19 population. Testing this hypothesis will require a focused research program.

There will never be a medical justification for smoking tobacco or using smoke-cured, tobacco products. Nevertheless, a recent non-peer-reviewed meta-analysis suggests that smoking may reduce the risk of hospitalization with COVID$1{ }^{[1]}$. An early study of COVID-19 patients in Wuhan reported a value of $6 \%$ for the prevalence of smoking in 78 patients ${ }^{[2]}$. More recent (April, 2020), peer-reviewed studies report that Chinese and American patient populations hospitalized for COVID-19 present a prevalence for smoking that is markedly lower than (or insignificantly different from) that of the surrounding population ${ }^{[2][3][4]}$. A non-peer-reviewed paper makes similar claims about French COVID-19 
patients $^{[5]}$. In the meta-analysis cited above ${ }^{[1]}$, values of smoking prevalence reported for Chinese COVID-19 patients range from $1.4 \%$ to $12.6 \%$, with an overall prevalence of $6.5 \%$ in a population of 5960 patients (13 studies). In contrast, smoking prevalence for the Chinese population at large is estimated to be $26.65 \%$. Thus, Chinese smokers appear to be less frequently counted among COVID-19 patients than among the population at large. Similar findings are reported for American and French COVID-19 patients.

Do these data mean that smoking protects smokers from being hospitalized with COVID-19? Frankly, it seems unlikely that smoking prevents viral exposure or viral entry into the respiratory system. However, it is conceivable that, once the virus enters the airways, a cellular / pharmacological mechanism allows nicotine or smoking to reduce the initial probability, or severity, of disease progression. Our research group has spent decades conducting research on the molecular actions of nicotine. It is of course possible, and perhaps eventually testable in animal models, that events downstream from interactions between nicotine and nicotinic acetylcholine receptors (nAChRs) (including $\alpha 4 \beta 2, \alpha 3 \beta 4$, and/or $\alpha 7$ nAChRs) exert protection against COVID-19. These mechanisms could include changes in angiotensin converting enzyme 2 (ACE2) levels ${ }^{[6][7]}$ and/or suppression of inflammatory pathways ${ }^{[8][9]}$. Treatment with nicotine patches, nicotine gum, or even electronic nicotine delivery systems (ENDSs) could provide nicotine without the other harmful effects of smoking.

Yet, once COVID-19 patients are admitted to the hospital, any hypothesized protection apparently disappears. There is no evidence that smokers admitted to hospitals present less severe COVID-19 symptoms than non-smokers. Two metaanalyses show that smoking either increases, or does not affect, the odds ratio for finding smokers in the severely ill COVID-19 population. In fact, an extensive meta-analysis of the association between smoking and COVID-19 severity reports an overall two-fold increase in the odds ratio for finding smokers among the severe COVID-19 patients, and the increase is significant ${ }^{[2]}$. Inclusion in the severe COVID-19 patient group was based on the American Thoracic Society guidelines for community-acquired pneumonia ${ }^{[4]}$. A less extensive meta-analysis reports no significant association between active smoking and COVID-19 severity ${ }^{[10]}$. Thus, smoking "protection"-if it exists--appears to apply only to nonhospitalized individuals. These surprising results call for confirmation and mechanistic explanations.

Other than the cellular / pharmacological "protection" mechanisms briefly described above, one can envision at least two straightforward, alternative explanations for the reduced prevalence of smokers in the COVID-19 patient population. The first explanation is systematic bias in identifying smokers in the hospital patient population, compared to the general population. The second is "reverse causation".

Hospital admissions staff could systematically underestimate the number of smokers among COVID-19 patient because (1) patients fail to inform hospital staff of their smoking history or (2) obtaining accurate medical histories under emergency conditions is problematic. For example, urine testing of hospitalized patients in Australia for the nicotine metabolite cotinine revealed that the admissions staff under-counted active or previous smokers by $37 \%$ because patients failed to accurately inform staff about their smoking behavior ${ }^{[11]}$. Previous studies by survey methodologists show that face-to-face interviews can introduce biases in certain types of attitudinal and behavioral questions not present in selfcompletion interviews ${ }^{[12][13]}$. Subjects in face-to-face interviews may be unwilling to admit socially undesirable behavior 
and attitudes under direct questioning ${ }^{[14]}$. In contrast to face-to-face interviews, government agencies and academic researchers find no evidence for strong social desirability bias in self-reported surveys of smoking behavior ${ }^{[15][16][17]}$. Considering the conditions under which COVID-19 patients may be interviewed during hospital admission, there are justifiable reasons to suspect the accuracy of smoking histories obtained from these interviews. COVID-19 patients may be quite ill with severe respiratory symptoms, including coughing, trouble breathing, and shortness of breath. They are probably wearing a mask and will likely encounter health care professionals who are themselves masked, gloved, and wearing face protection, impeding both verbal and visual doctor-patient communication. Patients may find it difficult to answer questions, wish to hurry through the interview process, and downplay potentially risky behaviors such as smoking. Even well-intentioned health care professionals may not conduct thorough interviews because of stress and overwork. For example, in a large Chinese study (Table 3 of ${ }^{[18]}$ ), patients with unknown smoking status were lumped together with non-smokers in a single non-smoking category (Never/unknown). Thus, failure to correctly identify active and previous smokers at the time of hospital admission could reduce estimates of smoking prevalence among COVID-19 patients, relative to the general population.

A second alternative explanation is "reverse causation". Patients hospitalized with COVID-19 may be simply less prone to use tobacco. A potentially robust "reverse causation" hypothesis for reduced prevalence of smokers in the COVID-19 population is the enrichment of patients in that population with serious comorbidities that motivates them to quit, or avoid, smoking. That is, COVID-19 patients with comorbidities could be less prone to smoke. We call this explanation the "smoking cessation" hypothesis. Antismoking campaigns have effectively reduced smoking in recent decades. The smoking rate among American adults was $42-45 \%$ in the mid-20th Century. It is now 14\% ${ }^{[19]}$. Antismoking campaigns emphasize that individuals with underlying medical conditions exacerbated by smoking-diabetes, cardiovascular disease, obesity, cancer--should quit. Consequently, individuals with these conditions may smoke less (perhaps by several fold) or cease smoking all together. Several important risk factors for tobacco-related disease-hypertension, obesity, and diabetes--overlap substantially with COVID-19 risk factors. It seems likely that such high-risk individuals have altered their behavior to smoke less or quit altogether. If so, this population presents a lower smoking prevalence than the general population. If individuals who quit smoking because of tobacco-related disease contract COVID-19 and become hospitalized, their low smoking prevalence is expected to reduce that of COVID-19 patients in general because patients with such comorbidities comprise a major fraction of the COVID-19 population [20]. In other words, we suggest that successful intervention in the high-risk groups during the pre-COVID-19 years, by antismoking campaigns, medical providers, and family members, has reduced smoking prevalence in the COVID-19 population. Also, many nursing homes prohibit smoking. Nursing facilities contribute large numbers of patients to the COVID-19 population. We judge that the "smoking cessation" mechanism may account for a significant fraction of the reduced prevalence of smokers in the COVID-19 population. Testing this hypothesis will require a focused research program. Our judgement that "protection" is unlikely derives, in part, from considering the history of research on the negative correlation between smoking history and the lifetime risk of Parkinson's disease (PD, see Note 1). Despite repeated attempts, there are no reports of successful efforts to ameliorate Parkinson's disease with nicotine patches or other nicotine replacement therapy. 


\section{Testing the "Smoking Cessation" vs "Protection" Hypotheses}

Medical histories and records taken during hospital admission provide the currently available epidemiological data. Oral histories of patient lifestyle are likely to be rushed or incomplete during a pandemic. In contrast, smoking data from smoking prevention and cessation programs collected by health maintenance organizations (HMOs) are reliable ${ }^{[21]}$. Subjects typically enter these programs because they have a high risk of tobacco-related disease and wish to reduce their

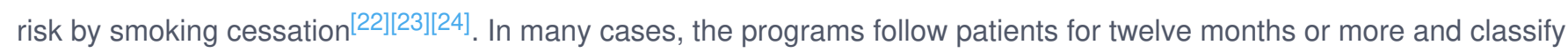
them as "successful" and "unsuccessful" quitters. Smoking status and participation in smoking cessation programs are now part of electronic medical records, which allow HMOs to routinely determine whether subjects have become COVID19 patients.

Smoking could both increase the probability of COVID-19 hospitalization by exacerbating smoking-related diseases and exert a protective effect by a still unidentified mechanism. Mindful of this complication, preliminary studies could test the contrasting predictions made by the "smoking cessation" and "protection" hypotheses. For individuals without pre-existing medical conditions (such as hypertension, chronic obstructive pulmonary disease (COPD), or diabetes) prior to hospitalization with COVID-19, the predictions are straightforward. The "smoking cessation" hypothesis predicts that successful (ex-smokers) and unsuccessful quitters (active smokers) will present a similar incidence of COVID-19 hospitalizations during the pandemic. In contrast, the "protection" hypothesis predicts that, in general, active smokers will present a lower incidence. The predictions become more complicated for individuals with smoking-related diseases prior to COVID-19 hospitalization because continued smoking is expected to exacerbate these diseases. For individuals with smoking-related diseases, the "smoking cessation" hypothesis predicts that successful quitters will present a similar, or lower, incidence of COVID-19 hospitalization compared to unsuccessful ones because failure to quit could exacerbate COVID-19 comorbidities. Predictions for the "protection" hypothesis depend on whether the deleterious effects of continued smoking on COVID-19 comorbidities outweigh its protective effects. Thus, predictions in this case are difficult to make a priori. Such an analysis will make an important contribution to mechanistic discussions about the low smoking prevalence of COVID-19 patients.

Predictions are also less clear for patients who have stopped smoking because they take varenicline-a nicotinic partial agonist—or because they use ENDSs. Data on these subjects will increase the interest of the studies. Confounding variables include the possibility that smokers put their hand near their mouths more often than non-smokers, increasing the likelihood of infection.

Finally, it will also be important to know how many COVID-19 patients stopped smoking when they felt the irritation of initial COVID-19 symptoms. As HMOs treat more COVID-19 patients, and increasingly test members for SARS-CoV-2 infection and antibodies, their databases will become troves of information about the interactions between lifestyle choices, such as smoking, and COVID-19. It will eventually be possible to query all tested members, including those who have not been hospitalized and those who stopped smoking voluntarily.

This commentary emphasizes the knowledge to be gained by analyzing smoking behavior in depth before and during the 
COVID-19 pandemic. If, over the upcoming years, the "smoking cessation" hypothesis increases support for health maintenance organizations, their antismoking campaigns deserve congratulations. Their successful efforts at prevention and cessation will have mitigated harm among individuals at risk for tobacco-related disease and possibly COVID-19.

Funding

This work was supported by the National Institutes of Health (DA0433829 to H.A.L.; ES010544, ES030175, and AG060908 to B.R.; DA046122 to S.G.), the Tobacco-Related Disease Research Program (27IP-0057 to H.A.L.; 29IR0445 to D.A.D.; 27FP-002 to A.L.N.) and the John Randolph Haynes and Dora Haynes Foundation (to R.M.A.)

Declaration of Interests

None declared.

Acknowledgements

We thank Michael H. Kanter (Kaiser Permanente Medical School) and Nora Volkow (NIDA) for comments and suggestions.

\section{Note 1.}

More than 70 studies, beginning in $1959^{25]}$, report a negative correlation between smoking and PD. These studies are both case-control and cohort. The overall average odds ratio for the PD effect is 2; the odds of finding smokers in the PD patient population are half that of finding them in the PD-free controls.

Data with animal models and cultured dopaminergic neurons further suggest that nicotine may underlie this negative correlation ${ }^{[26]}$. A plausible biological mechanism proposes that the interaction between nicotine and nicotinic acetylcholine receptors in the endoplasmic reticulum (ER) reduces ER stress and the unfolded protein response ${ }^{[27]}$ which, in turn, reduce the risk of PD.

Alternatively, "reverse causality" could explain this negative correlation. Anecdotally, neurologists report that early-stage PD patients stop smoking, even though they display few or no minor motor symptoms. Mechanistically, this tendency could be explained by damage to dopaminergic neurons during the prodromal stage, so that the prodromal patient derives less reward from activation of the nicotinic receptors on those neurons ${ }^{[28][29]}$. Regardless of the "reverse causality" mechanism, the result is that, several years later, when patients are diagnosed with PD, the PD population has a dearth of smokers and the non-PD population is comparatively enriched in smokers. This hypothesis postulates that the apparent neuroprotective effect of smoking occurs because PD patients are less likely to smoke-not because smokers are 
protected.

A "reverse causality" mechanism for COVID-19 based on patient behavior (COVID-19 patients with severe co-morbidities smoke less, rather than that smokers are protected) is analogous to that for PD.

\section{References}

1. a, barsalinos K, Barbouni A, Niaura R. Smoking, vaping and hospitalization for COVID-19. Qeios. 2020.

2. a, b, c Liu W, Tao ZW, Lei W, et al. Analysis of factors associated with disease outcomes in hospitalized patients with 2019 novel coronavirus disease. Chin Med J (Engl). 2020.

3. 'CDC_COVID-19_Response_Team. Preliminary Estimates of the Prevalence of Selected Underlying Health Conditions Among Patients with Coronavirus Disease 2019 - United States, February 12-March 28, 2020. Morbidity and Mortality Weekly Report (MMWR) 2020; https://www.cdc.gov/mmwr/volumes/69/wr/mm6913e2.htm. Accessed 25 April 2020.

4. ${ }^{a}$, buan WJ, Ni ZY, Hu Y, et al. Clinical Characteristics of Coronavirus Disease 2019 in China. N Engl J Med. 2020.

5. 'Miyara M, Tubach F, POURCHER V, Morelot-Panzini C, Pernet C, Haroche J. Low incidence of daily active tobacco smoking in patients with symptomatic COVID-19. Qeios. 2020.

6. 'Oakes JM, Fuchs RM, Gardner JD, Lazartigues E, Yue X. Nicotine and the renin-angiotensin system. Am J Physiol Regul Integr Comp Physiol. 2018;315(5):R895-r906.

7. 'McCray PB, Jr., Pewe L, Wohlford-Lenane C, et al. Lethal infection of K18-hACE2 mice infected with severe acute respiratory syndrome coronavirus. J Virol. 2007;81(2):813-821.

8. 'Rosas-Ballina M, Tracey KJ. The neurology of the immune system: neural reflexes regulate immunity. Neuron. 2009;64(1):28-32.

9. 'Gahring LC, Myers EJ, Dunn DM, Weiss RB, Rogers SW. Lung epithelial response to cigarette smoke and modulation by the nicotinic $\alpha 7$ receptor. PLoS One. 2017;12(11):e0187773.

10. 'Lippi G, Henry BM. Active smoking is not associated with severity of coronavirus disease 2019 (COVID-19). Eur J Intern Med. 2020;75:107-108.

11. 'Schofield PE, Hill DJ. How accurate is in-patient smoking status data collected by hospital admissions staff? Aust N Z J Public Health. 1999;23(6):654-656.

12. 'Atkeson LR, Adams AN, Alvarez RM. Nonresponse and Mode Effects in Self- and Interviewer-Administered Surveys. Polit. Anal. 2014;22(3):304-320.

13. ^Abrajano M, Alvarez RM. Answering Questions About Race: How Racial and Ethnic Identities Influence Survey Response. Am. Polit. Res. 2019;47(2):250-274.

14. ^Tourangeau R, Yan T. Sensitive questions in surveys. Psychol. Bull. 2007;133(5):859-883.

15. 'Patrick DL, Cheadle A, Thompson DC, Diehr P, Koepsell T, Kinne S. The validity of self-reported smoking: a review and meta-analysis. Am J Public Health. 1994;84(7):1086-1093. 
16. ^Yeager DS, Krosnick JA. The validity of self-reported nicotine product use in the 2001-2008 National Health and Nutrition Examination Survey. Med Care. 2010;48(12):1128-1132.

17. ^Persoskie A, Nelson WL. Just blowing smoke? Social desirability and reporting of intentions to quit smoking. Nicotine Tob Res. 2013;15(12):2088-2093.

18. 'Guan W-j, Liang W-h, Zhao Y, et al. Comorbidity and its impact on 1590 patients with Covid-19 in China: $A$ Nationwide Analysis. European Respiratory Journal. 2020:2000547.

19. ' Creamer MR, Wang TW, Babb S, et al. Tobacco Product Use and Cessation Indicators Among Adults - United States, 2018. MMWR Morb Mortal Wkly Rep. 2019;68(45):1013-1019.

20. ^Richardson S, Hirsch JS, Narasimhan M, et al. Presenting Characteristics, Comorbidities, and Outcomes Among 5700 Patients Hospitalized With COVID-19 in the New York City Area. Jama. 2020.

21. ^Young-Wolff KC, Fogelberg R, Preston PG. Implementing a Multifaceted Perioperative Smoking Cessation Intervention in a Large Healthcare System. Nicotine Tob Res. 2020;22(3):452-453.

22. ^Raz DJ, Wu G, Nelson RA, et al. Perceptions and Utilization of Lung Cancer Screening Among Smokers Enrolled in a Tobacco Cessation Program. Clin Lung Cancer. 2019;20(1):e115-e122.

23. `Schmittdiel JA, Cunningham SA, Adams SR, Nielsen J, Ali MK. Influence of a New Diabetes Diagnosis on the Health Behaviors of the Patient's Partner. Ann Fam Med. 2018;16(4):290-295.

24. ^Kaiser EG, Prochaska JJ, Kendra MS. Tobacco Cessation in Oncology Care. Oncology. 2018;95(3):129-137.

25. ^Dorn HF. Tobacco consumption and mortality from cancer and other diseases. Public Health Rep. 1959;74(7):581593.

26. `Quik M. Smoking, nicotine and Parkinson's disease. Trends Neurosci. 2004;27(9):561-568.

27. `Srinivasan R, Henley BM, Henderson BJ, et al. Smoking-Relevant Nicotine Concentration Attenuates the Unfolded Protein Response in Dopaminergic Neurons. J Neurosci. 2016;36(1):65-79.

28. ^Ritz B, Lee PC, Lassen CF, Arah OA. Parkinson disease and smoking revisited: ease of quitting is an early sign of the disease. Neurology. 2014;83(16):1396-1402.

29. ^Chuang YH, Paul KC, Sinsheimer JS, Bronstein JM, Bordelon YM, Ritz B. Genetic variants in nicotinic receptors and smoking cessation in Parkinson's disease. Parkinsonism Relat Disord. 2019;62:57-61. 